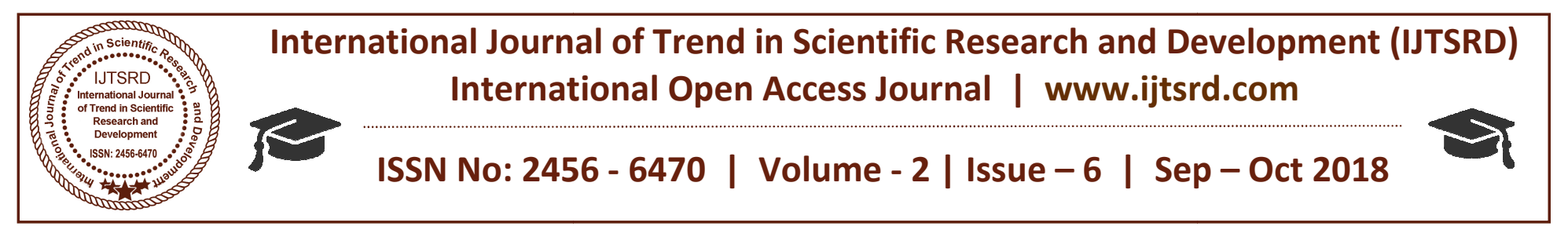

\title{
Modified Low-Energy Adaptive Clustering Hierarchy (M-LEACH) Protocol For WSN
}

\author{
Aditi Beohar ${ }^{1}$, Prof. Pankaj Sahu ${ }^{2}$, Prof. Rajender Singh Yadav ${ }^{2}$ \\ ${ }^{1}$ Research Scholar, ${ }^{2}$ Assistant Professor \\ Department of ECE, GGITS, Jabalpur, Madhya Pradesh, India
}

\begin{abstract}
In this work, M-LEACH (Modified Distributed Energy Efficient Clustering) protocol, a new variant of LEACH is proposed. The proposed M-LEACH is designed for three different segregations for the nodes to elongate the stability \& lifetime of the network. Hence, it increases the heterogeneity \& energy level of the network. In LEACH, amplification energy is set same for all kinds of transmissions. Using low energy level for intra cluster transmissions with respect to cluster head to BS transmission leads in saving much amount of energy. Moreover, multi power levels also reduce the packet drop ratio, collisions and/ or interference for other signals. The proposed $\mathrm{M}$ LEACH outperforms in all other existing $\mathrm{LEACH}$ variants when compared for FND, HND \& LND.
\end{abstract}

Keyword: LEACH, WSN, Routing Protocol, $M-$ LEACH, Efficient Clustering, Cluster Head, FND, $H N D, L N D$

\section{INTRODUCTION}

Everybody in current scenario need immediate information in every aspect of our lives. For achieving this need, several networks are designed to pass information. Ad-Hoc networks give infrastructure-less communication. Multi hop networks were designed to give more liberty of movement. In case of wireless sensor networks, that device normally is termed as a sensor, node or mote and it has its own limitations i.e. it must be capable of sensing, processing and transmitting/ receiving. Each node hence also require a power source to perform all these operations. Considering applications of wireless sensor networks, installing a battery on each sensor node is a better solution. Furthermore, limiting use of power is one of the key challenges in wireless sensor networks. These batteries must be smart enough to give a node maximum life despite of being tiny sized.
Any technology that is in process of its development, give a lot of challenges. In the same way, wireless sensor networks do. Sensing, computing and transcieving by tiny sized sensors with power constraint is not a simple thing. Hence this is the major concern for scientists and researchers. To optimize node's life time, we need to focus on such algorithms, protocols and physical circuitries that can make maximum out of limited power source.

In any network especially wireless multi hop networks, for efficient performance; its protocols must be very efficient. Numerous protocols are developed that address power problem in sensor networks. Most prominent routing algorithms can be categorized into three types' i.e. direct transmission algorithms, hop to hop transmission algorithms and cluster based algorithms.

Another problem that persists is to handle bulk of information sensed and passed over by every node of a network. (A WSN may consist of thousands of nodes). For that data aggregation and data fusion algorithms work, however there is always a room for betterment. In an efficient wireless sensor network, we need efficient routing protocol that has low routing overhead and well organized data aggregation mechanisms to increase good put of network and to save limited power of sensor node.

In next sections, we discuss about the work done on cluster based routing of wireless sensor networks along with areas which need modifications to enhance efficiency. Later, some modifications are made in one of most prominent routing protocol. Finally, experiments along with comparisons are made and discussed briefly. 


\section{RELATED WORK}

Manufacturing of cheap wireless sensor nodes having sufficient computation and transmitting/ receiving powers are available now. Hence hundreds of nodes can be deployed in a network for any required application. These sensor nodes have a limited power which must be utilized in very precise manner to increase node's life. No doubt efficient circuit is necessary for efficient use of energy, however, routing protocol running on the network plays a vital role in bandwidth consumption, security and energy conservations as well (considering WSN's).

To cover with these constraints, initially direct transmission approach was discussed [1]. In direct transmission, a node sense data from its environment and transmits it straight to base station. This method, no doubt, ensures data security however; on the other hand we have to compromise on node's life time due to excessive power consumption (if BS is far away). Hence, using direct transmission technique, nodes that are far away from BS die early as they require more power to propagate their signal, making a portion of field vacant for sensing.

To solve this problem, minimum transmission energy (MTE) emerged. In this technique, data is transmitted to base stations via multi hop. This gives birth to almost same problem we faced in direct transmission. Difference is only this that in minimum transmission energy algorithm, far away nodes remain alive longer with respect to the nodes nearer to BS. Reason behind early expiry of nearer nodes is routing of all data traffic to base station. Moreover, transmitting bulk of sensed data from each node use much energy. To overcome this problem, concept of Directed Diffusion was introduced that discuss data processing and dissemination [2]. Estrin ET. Al [3] worked on an hierarchical clustering mechanism dealing with asymmetric communication for power saving in sensor nodes. Jiang et.al presented a cluster based routing protocol (CBRP) [4]. According to this mechanism, all participating nodes of network are distributed in 2-hop cluster. Though this protocol is not much energy efficient for wireless sensor nodes however, it gives way to hierarchical clustering algorithms. Clustering for energy conservation is proven as efficient mechanism for wireless sensor networks $[5,6]$. When a sensor network is deployed, nodes establish clusters and nominate one node from each cluster as a cluster head. These cluster head nodes are responsible for receiving data from other nodes of cluster, do data aggregation/ fusion of received data and transmit it to base station. In this way, bandwidth consumption and life time of network is optimized [7]. In [8] authors give concept of inter cluster communication. They prove that regardless of transmitting fused data direct from cluster head to base station, if data is transmitted in multiple hopes i.e. from one cluster head to another and finally to base station, it would further enhance network life time.

M. Tahir ET. $A l$ [21] introduces link quality metric to divide a network into three logical portions resulting in lower routing overhead. Authors of [22] preserve energy in WSN's by differentiating idle and operational mode of a sensor node.

Authors of $[9,10]$ states that nodes having high initial energy will be selected as cluster heads (in case of heterogeneous sensor networks). While according [11, 12, and 13] any node that lie within network can be elected as a cluster head. Stable Election Protocol (SEP) gives weighted probability to each node of becoming a cluster head [11]. In DEEC [12] existing energy in node is election criteria of a node to become a cluster head.

LEACH [1], TEEN [14], SEP [11], DEEC [12] and PEGASIS [15] are prominent routing techniques for wireless sensor networks. Main procedure of electing a cluster head was given by LEACH and that is further enhanced by SEP and DEEC. TEEN introduces the concept of thresholds that gives good results in network life time by showing reactive nature. These thresholds can be implemented in any routing protocol to enhance its performance with respect to utility or application. Considering LEACH, the algorithm is divided into three parts, i.e. advertising phase, Cluster Set up phase and Scheduling phase.

LEACH gives birth to many protocols. The procedures of this protocol are compact and well coped with homogeneous sensor environment. According to this protocol, for every round, new cluster head is elected and hence new cluster formation is required. This leads to unnecessary routing overhead resulting in excessive use of limited energy. If a cluster head has not utilized much of its energy during previous round, than there is probability that some low energy node may replace it as a cluster head in next cluster head election process. 
There is a need to limit change of cluster heads at every round considering residual energy of existing cluster head. Hence an efficient cluster head replacement algorithm is required to conserve energy.

In clustering protocols as LEACH, nodes use same amplification energy to transmit data regardless of distance between transmitter and receiver. To preserve energy, there should also be transmission mechanisms that specify required amplification energy for communicating with cluster head or base station. For example, transmitting a packet to cluster head with same amplification power level as required by a node located at farthest end of network to base station results in wastage of energy, One solution can be having global knowledge of network and then nodes decide how much they need to amplify signal. Locating and calculating distances with in full network topology needs lot of routing and so, this approach do not work for saving energy. To solve above mentioned problems, we propose two mechanismsi. E. efficient cluster head replacement and dual transmitting power levels.

\section{PROPOSEDMETHODOLOGY}

Our work is based on LEACH protocol that can be extended further for other protocols. Basically, we introduce two techniques to raise network life time and throughput. To understand our proposed scheme, we have to understand mechanism given by LEACH. This protocol changes the cluster head at every round and once a cluster head is formed, it will not get another chance for next $1 / p$ rounds. For every round, cluster heads are replaced and whole cluster formation process is undertaken. We, in this work, modify LEACH by introducing "efficient cluster head replacement scheme". It is a threshold in cluster head formation for very next round. If existing cluster has not spent much energy during its tenure and has more energy than required threshold, it will remain cluster head for the next round as well. This is how, energy wasted in routing packets for new cluster head and cluster formation can be saved. If cluster head has less energy than required threshold, it will be replaced according to LEACH algorithm. Besides limiting energy utilization in cluster formation, we also introduce two different levels of power to amplify signals according to nature of transmission. Basically there can be three modes of transmission in a cluster based network.

1. Intra Cluster Transmission

2. Inter Cluster Transmission

\section{Cluster Head To Base Station Transmission}

Intra Cluster Transmission deals with all the communication within a cluster i.e. cluster member's sense data and report sensed data to cluster head. The transmission/ reception between two clusters heads can be termed as inter cluster transmission while a cluster head transmitting its data straight to base station lies under the caption of cluster head to base station transmission.

Minimum amplification energy required for inter cluster or cluster head to BS communication and amplification energy required for intra cluster communication cannot be same. In LEACH, amplification energy is set same for all kinds of transmissions. Using low energy level for intra cluster transmissions with respect to cluster head to BS transmission leads in saving much amount of energy. Moreover, multi power levels also reduce the packet drop ratio, collisions and/ or interference for other signals. In this context, we assume that a cluster at maximum may spread into an area of $10 X 10 \mathrm{~m}^{2}$ in a field of $100 \times 100 \mathrm{~m}^{2}$. Energy that is enough to transmit at far ends of a field of $100 \times 100 \mathrm{~m}^{2}$ must be lowered 10 times for intra-cluster transmission. When a node act as a Cluster head, routing protocol informs it to use high power amplification and in next round, when that node becomes a cluster member, routing protocol switches it to low level power amplification. Finally, soft and hard threshold schemes are also implemented in M-LEACH that gives better results.

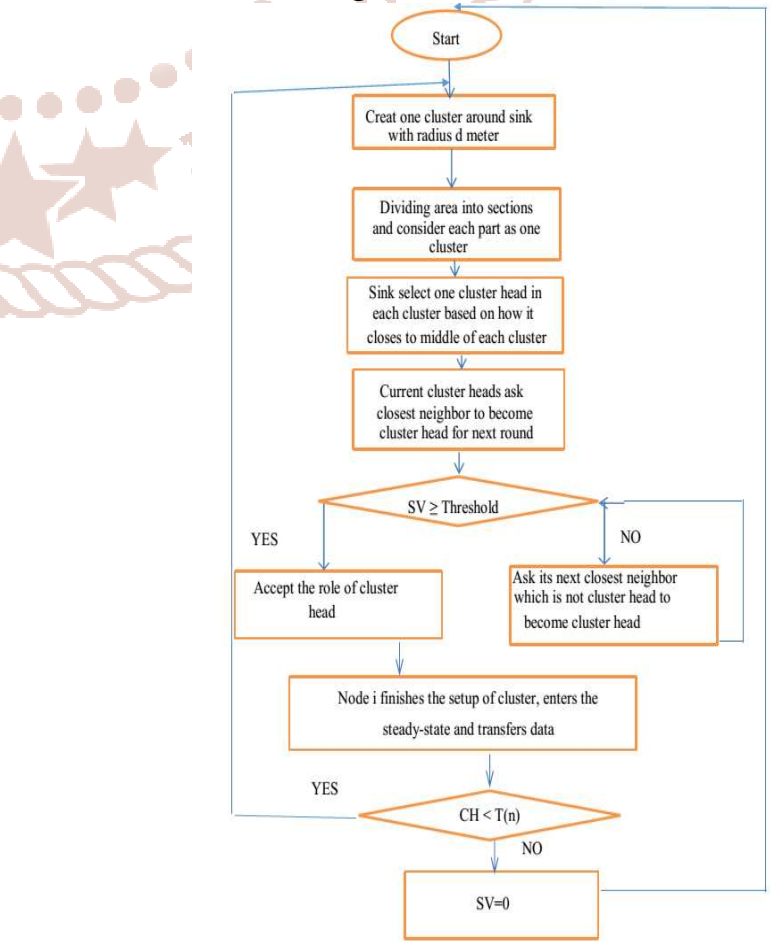

Figure 1: Proposed M-Leach Flow Chart 
International Journal of Trend in Scientific Research and Development (IJTSRD) ISSN: 2456-6470

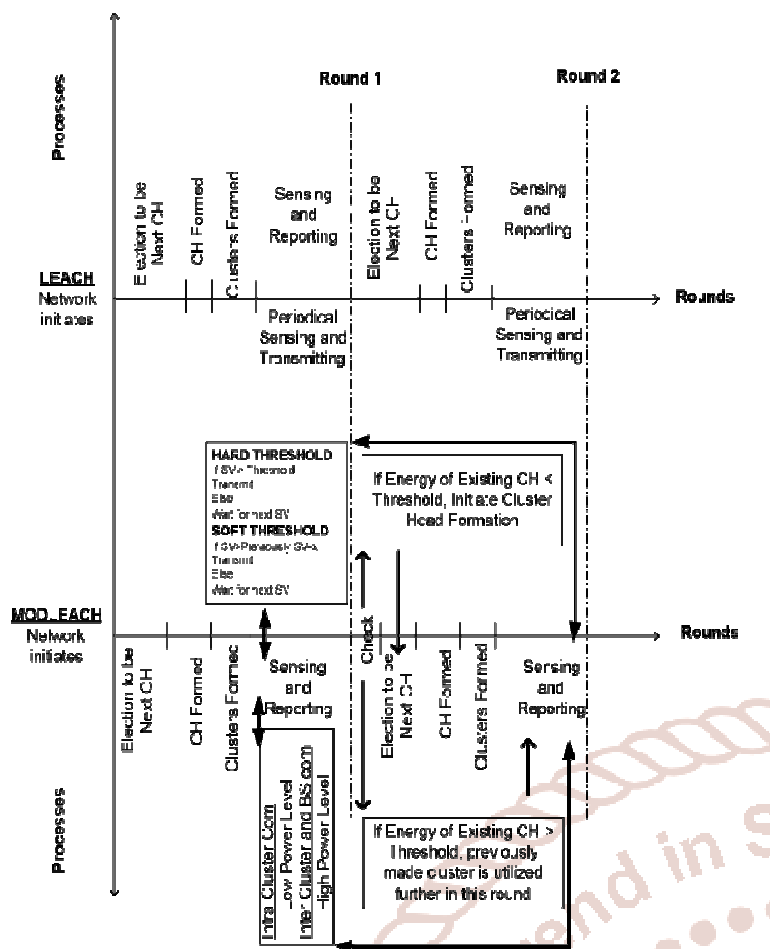

Figure 2: Proposed M-LEACH Functioning

\section{SIMULATION RESULTS \& DISCUSSION}

All the simulations are conducted using MATLAB (R2013b).For the simulation in MATLAB following parameters are taken as the benchmark:

\begin{tabular}{|l|c|}
\hline \multicolumn{1}{|c|}{ Network Parameters } & Value \\
\hline Network Field Size & $100 \times 100 \mathrm{~m}^{2}$ \\
\hline Number of Nodes & 100 \\
\hline Initial Energy of Sensor Nodes $\left(E_{o}\right)$ & $0.5 \mathrm{~J}$ \\
\hline Packet / Message Size & $4000 \mathrm{bits}$ \\
\hline Transceiver idle state energy consumption $\left(E_{\text {elec }}\right)$ & $50 \mathrm{~nJ} / \mathrm{bit}$ \\
\hline Data Aggregation/ Fusion Energy consumption $\left(E_{f s}\right)$ & $10 \mathrm{~nJ} / \mathrm{bit} / \mathrm{report}$ \\
\hline Amplification Energy (Cluster to BS) $\left(E_{\text {amp }}\right)$ & $0.0013 \mathrm{pJ} / \mathrm{bit} / \mathrm{m} 2$ \\
\hline Energy Consumption of Data Gathering Cluster Head $\left(E_{D A}\right)$ & $5 \mathrm{~nJ} / \mathrm{bit} / \mathrm{signal}$ \\
\hline Threshold Distance $\left(d_{o}\right)$ & $70 \mathrm{~m}$ \\
\hline$P_{\text {opt }}$ & 0.1 \\
\hline
\end{tabular}

Table 1: Parameters for simulation of our proposed M-LEACH implementation

\section{IV.1 Network Life Time / Dead \& Alive Nodes}

To examine the performance of wireless sensor networks some characterization parameters are generally used. These parameters are related to number of nodes, alive or dead \& network life time span. Some of them are:

\section{FND (First Node Dead):}

The time span from start to when the first node dead is called FND (First Node Dead).

\section{HND (Half number of Nodes Dead):}

What's more, the round when half of the nodes die is called HND (Half number of Nodes Dead).

\section{LND (Last Node Dead):}

Another measure is LND (Last Node Dead), which is the time span from the time zero to when there is no a live node in the network.

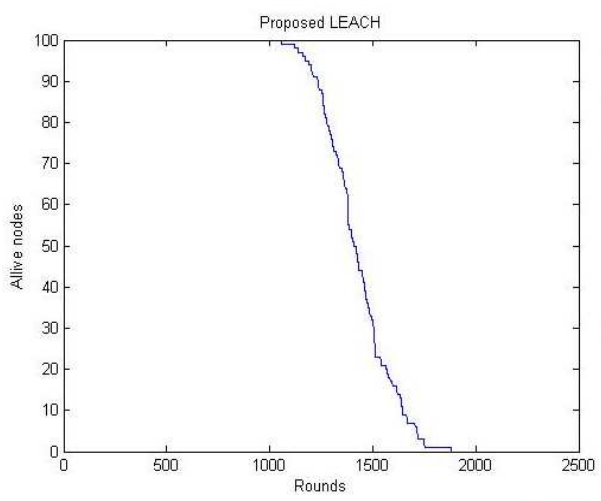

Figure 3: Number of Alive Nodes vs Rounds

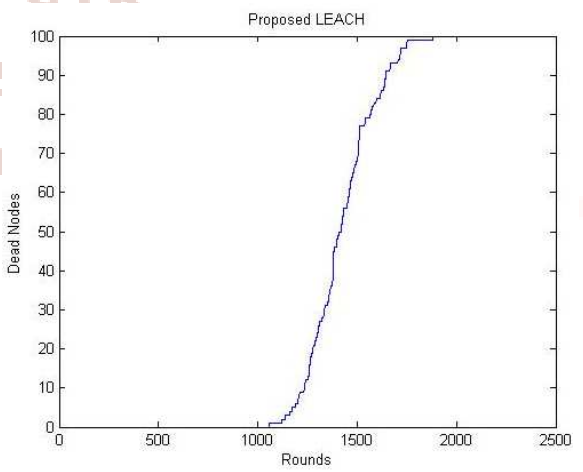

Figure 4: Number of Dead Nodes vs Rounds

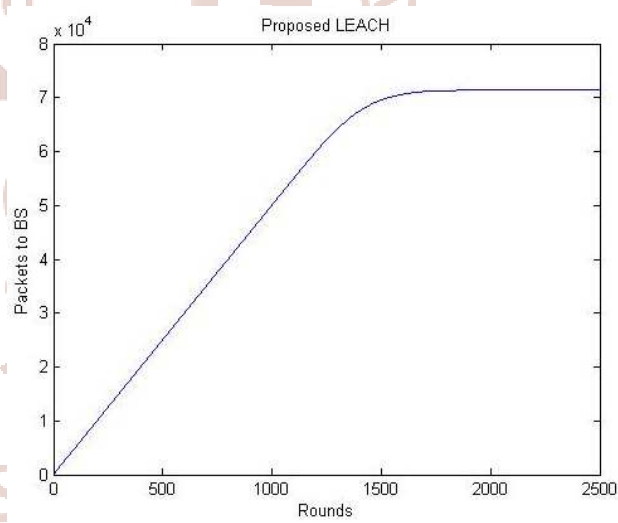

Figure 5: Packet sent to Base Station vs. Rounds

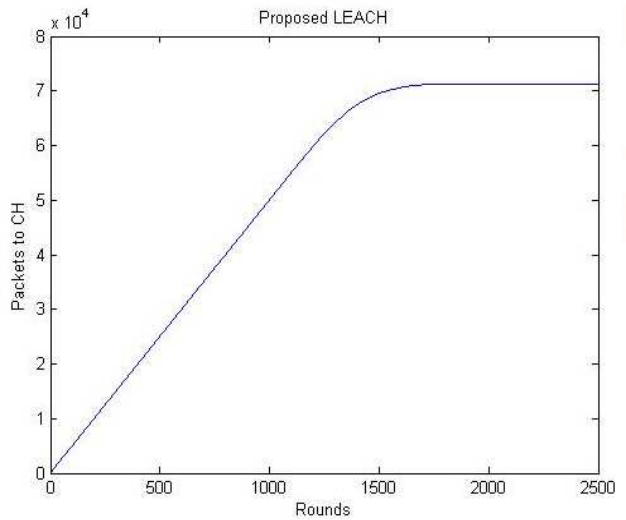

Figure 6: Packet sent to Cluster Head vs. Rounds 


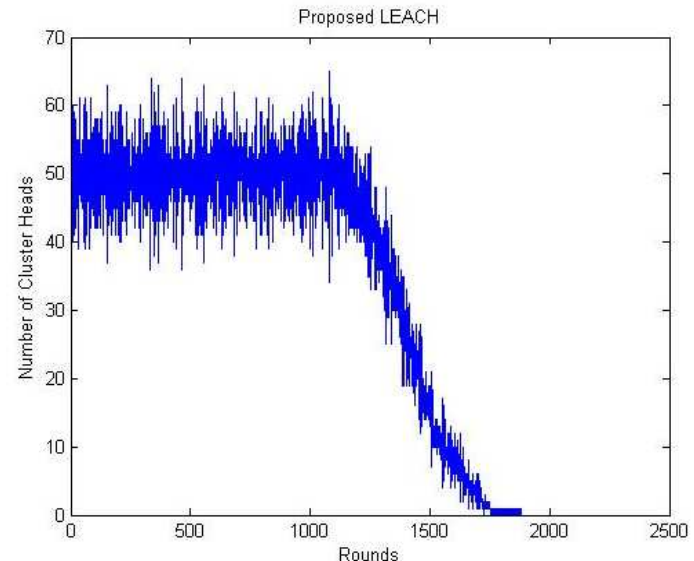

Figure 7: Number of Cluster Heads vs Rounds

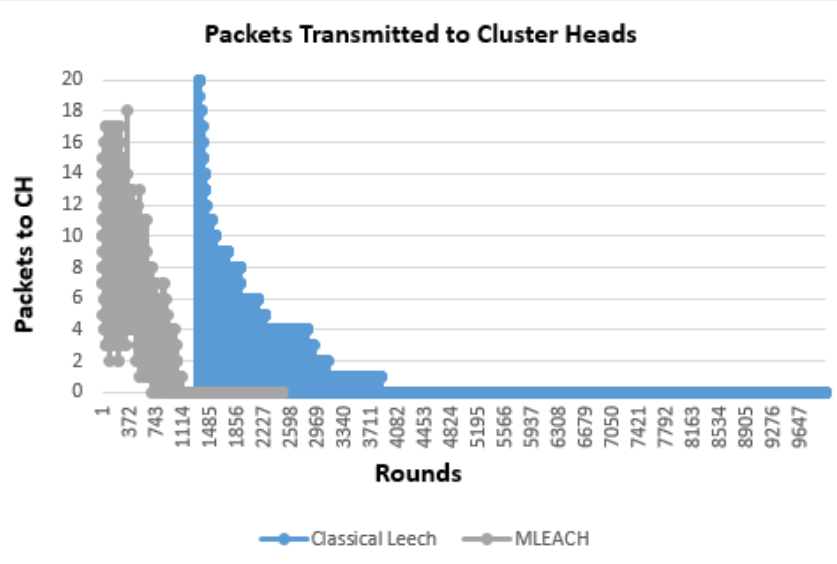

Figure 10: Comparison of Packets Transmitted to $\mathrm{CH}$

\begin{tabular}{|c|c|c|c|}
\hline Parameters & FND & HND & LND \\
\hline LEACH & 200 & 430 & 710 \\
\hline T-LEACH [2] & 170 & 580 & 790 \\
\hline MT-CHR [2] & 330 & 770 & 910 \\
\hline C-DTB-CHR [2] & 410 & 890 & 1170 \\
\hline DEEC [3] & 870 & 1130 & 1270 \\
\hline CH-LEACH [3] & 750 & 1500 & 1700 \\
\hline MS-LEACH [4] & 946 & 1120 & 1190 \\
\hline IDE-LEACH [4] & 1007 & 1122 & 1200 \\
\hline Proposed M-LEACH & 1020 & 1420 & 1820 \\
\hline
\end{tabular}

Table 3: Simulation Result Comparison

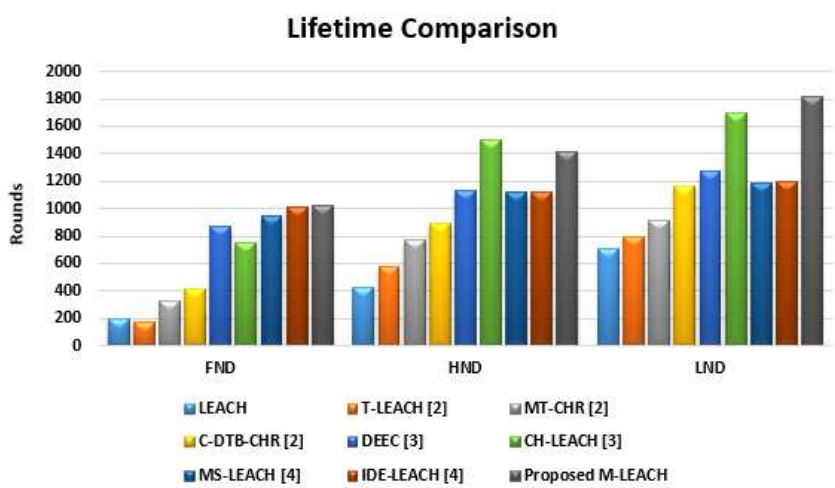

Figure 11: Comparison of Proposed M-LEACH with other Variants

\section{CONCLUSION}

In this work, we give a brief discussion on emergence of cluster based routing in wireless sensor networks. We also propose M-LEACH, a new variant of LEACH that can further be utilized in other clustering routing protocols for better efficiency. In this work, M-LEACH (Modified Distributed Energy Efficient Clustering) protocol, a new variant of $\mathrm{LEACH}$ is proposed. The proposed M-LEACH is designed for three different segregations for the nodes to elongate the stability \&lifetime of the network. Hence, it

Figure 9: Comparison of Packets Transmitted To Base Station 
increases the heterogeneity \& energy level of the network. In LEACH, amplification energy is set same for all kinds of transmissions. Using low energy level for intra cluster transmissions with respect to cluster head to BS transmission leads in saving much amount of energy. Moreover, multi power levels also reduce the packet drop ratio, collisions and/ or interference for other signals. The proposed M-LEACH outperforms in all other existing LEACH variants when compared for FND, HND \& LND.

\section{REFERENCES}

1. Shweta V. et-al, "Variants of LEACH Routing Protocol in WSN: A Comparative Analysis", 8th IEEE International Conference on Cloud Computing, Data Science \& Engineering (Confluence), 2018.

2. Khalid A.D. et-al, "A New Cluster Head Replacement Protocol for Wireless Sensor Networks", IEEE European Conference on Electrical Engineering and Computer Science (EECS), 2017.

3. WalidAbushiba et-al, "An Energy Efficient and Adaptive Clustering for Wireless Sensor Network (CH- LEACH) using LEACH Protocol", 13th IEEE International Computer Engineering Conference (ICENCO), 2017.

4. Shweta Gupta et-al, "Improved Distance Energy Based LEACH Protocol for Cluster Head Election in Wireless Sensor Networks", 4th IEEE International Conference on Signal Processing, Computing and Control (ISPCC 2k17), 2017.

5. L.K. Suresh Kumar et-al, "Comparative Study of LEACH and EECDA Protocols", IEEE International Conference on Power, Control, Signals and Instrumentation Engineering (ICPCSI), 2017.

6. Korhan C. et-al, "Extending the Lifetime of WSNs with Maximum Energy Selection Algorithm (MESA)", IEEE International Black Sea Conference on Communications and Networking, 2016.

7. Yuan Zhou et-al, "Clustering Hierarchy Protocol in Wireless Sensor Networks Using an Improved PSO Algorithm", IEEE ACCESS, 2016.

8. Saad A. Alharthi et-al, "Threshold Sensitive Heterogeneous LEACH Protocol for Wireless Sensor Networks", 24th Telecommunications forum TELFOR, IEEE, 2016.
9. Alka Singh et-al, "Energy Efficient Routing of WSN using Particle Swarm Optimization and VLeach Protocol", IEEE International Conference on Communication and Signal Processing, 2016.

10. Kushal B Y et-al, "Cluster Based Routing Protocol to Prolong Network Lifetime through Mobile Sink in WSN", IEEE International Conference on Recent Trends in Electronics Information Communication Technology, 2016.

11. M. Sajid et-al, "A new Linear Cluster Handling (LCH) Technique towards Energy Efficiency in Linear WSN's", 29th IEEE International Bhurban Conference on Advanced Information Networking and Applications, Pp. 389 - 393, 2015.

12. Dasari Raja et-al, "Performance Analysis of Selforganized Tree Based Energy Balance (STEB) Routing Protocol for WSN", IEEE International Conference on Communications and Signal Processing (ICCSP), 2015.

13. Mahmoud M. Salim, Hussein A. Elsayed, Salwa H. El Ramly, "PR-LEACH: Approach for balancing Energy Dissipation of LEACH protocol for Wireless Sensor Networks", 31st National Radio Science Conference (NRSC), IEEE, 2014.

14. TrianaMugiaRahayu, Sang-Gon Lee, Hoon-Jae Lee, "Survey on LEACH-based Security Protocols", 16th IEEE International Conference on Advanced Communication Technology (ICACT), Pyeongchang, 2014.

15. Asha Ahlawat, Vineeta Malik, "An Extended Vice-Cluster Selection Approach to Improve VLeach Protocol in WSN", 3rd IEEE International Conference on Advanced Computing \& Communication Technologies, 2013.

16. K. Latif, M. Jaffar, N. Javaid, M. N. Saqib, U. Qasim , Z. A. Khan, "Performance Analysis of Hierarchical Routing Protocols in Wireless Sensor Networks", 7th IEEE International Conference on Broadband, Wireless Computing, Communication and Applications, 2012.

17. Mohammed Abo-Zahhad, Sabah M. Ahmed, Nabil Sabor and Shigenobu Sasaki, "A New Energy-Efficient Adaptive Clustering Protocol Based on Genetic Algorithm for Improving the Lifetime and the Stable Period of Wireless Sensor Networks", International Journal of Energy, Information and Communications Vol.5, Issue 3 (2014), pp.47-72. 
18. S. K. Singh, M. P. Singh and D. K. Singh, "Routing Protocols in Wireless Sensor Networks A Survey", Int. Journal of Computer Science and Engineering Survey (IJCSES), vol. 1, no. 2, (2010) November, pp. 63-83.

19. S. A. Sayyadi, R. Senemar and H. Teimuri, "Elect Distance Efficient Cluster Heads for Improvement Performance of LEACH Protocol", 2nd International Conference on Computational Techniques and Artificial Intelligence (ICCTAI'2013), Dubai, (2013) March 17-18, pp. 179-183.

20. W. Heinzelman, A. Chandrakasan and $H$. Balakrishnan, "An Application-Specific Protocol Architecture for Wireless Micro sensor Networks," IEEE Transactions on Wireless Communications, vol. 1, no. 4, (2002) October, pp. 660-670.

21. F. Xiangning and S. Yulin, "Improvement on LEACH Protocol of Wireless Sensor Network", Conference on Sensor Technologies and Applications, Valencia, (2007) October 14-20, pp. 260-264.

22. V. Loscrì, G. Morabito and S. Marano, "A TwoLevel Hierarchy for Low-Energy Adaptive Clustering Hierarchy", IEEE 62nd Vehicular Technology Conference, (2005) September 25-28, pp. 1809-1813.

23. M. B. Young, A. Al-zou'bi, Y. Khamayseh and W. Mardini, "Improvement on LEACH Protocol of Wireless Sensor Network (VLEACH)", International Journal of Digital Content Technology and Its Applications, vol. 3, no. 2, (2009), pp. 132-136.

24. J. L. Liu and C. V. Ravishankar, "LEACH-GA: Genetic Algorithm-Based Energy-Efficient
Adaptive Clustering Protocol for Wireless Sensor Networks", Int. J. of Machine Learning and Computing, vol. 1, no. 1, (2011) April, pp. 79-85.

25. K. Go. Vijayvargiya and V. Shrivastava, "An Amend Implementation on LEACH protocol based on Energy Hierarchy", International Journal of Current Engineering and Technology, vol. 2, no. 4, (2012) December, pp. 427-431.

26. R. Halke and V. A. Kulkarni, "En-LEACH Routing Protocol for Wireless Sensor Network", International Journal of Engineering Research and Applications (IJERA), vol. 2, no. 4, (2012) August, pp. 2099-2102.

27. J. S. Brunda, B. S. Manjunath, B. R. Savitha and P. Ullas, "Energy Aware Threshold based Efficient Clustering (EATEC) for Wireless Sensor Network", International Journal of Computer Technology and Electronics Engineering (IJCTEE), vol. 2, no. 4, (2012) August, pp. 25-30.

28. W. Liu and L. Wang, "An improved algorithm based on LEACH protocol", Journal of Applied Mechanics and Materials, vol. 347, (2013), pp. 2725-2727.

29. K. Pawar, V. Pawar and T. Sharma, "Enhancement of LEACH Protocol Using Energy Heterogeneity Concept", International Journal of Emerging Trends and Technology in Computer Science (IJETTCS), vol. 2, no. 1, (2013) February, pp. 49-56.

30. P. Bakaraniya and S. Mehta, "K-LEACH: An improved LEACH Protocol for Lifetime Improvement in WSN", International Journal of Engineering Trends and Technology (IJETT), vol. 4, no. 5, (2013) May, pp. 1521-1526. 